\title{
Robust stationary distributed discord in Jordan-Wigner fermion system under perturbations of initial state
}

\author{
E.B.Fel'dman and A.I. Zenchuk \\ Institute of Problems of Chemical Physics, RAS, Chernogolovka, Moscow reg., 142432, Russia, \\ e-mail: efeldman@icp.ac.ru, zenchuk@itp.ac.ru
}

\begin{abstract}
We investigate the Jordan-Wigner fermion clusters with the stationary distributed pairwise quantum discord. Such clusters appear after the Jordan-Wigner transformation of a spin chain governed by the nearest-neighbor XY-Hamiltonian with the particular initial state having one polarized node. We show that the quantum discord stationarity in such systems is not destroyed by the "parasitic" polarization of at least two types. First type appears because the initial state with a single polarized node is hardly realizable experimentally, so that the low polarization of neighboring nodes must be taken into account. Second, the additional noise-polarization of all nodes is unavoidable. Although the stationarity may not be destroyed by perturbations of the above two types, the parasitic polarizations deform the distribution of the pairwise discord and may destroy the clusters of correlated fermions with equal pairwise discords. Such deformations are studied in this paper.
\end{abstract}

\section{Introduction}

The quantum correlations are responsible for the effective operation of quantum information devices having the essential advantages in comparison with their classical counterparts [1, 2, 3, 4, 15, 6, 17, 8, 9, 10, 11, 12, 13, 14. According to the current stand point, the total correlations in multi-particle system are described by mutual information and quantum correlations for both pure and mixed states are characterized by the quantum discord [2, 3, 4, 15, 10, 11, 12, 13, 14.

Studing the quantum correlations, it is important to choose the proper quantum system possessing the desirable properties and realizable in practice. In this regard we point on the chains of nuclear spins, which are suitable for realization of quantum registers and quantum devices transferring and manipulating the quantum information. It is challenging that the multiple quantum (MQ) NMR methods [15, 16] allow us to construct experimentally the XYinteracting spin chains. Moreover, using the NMR method, it is possible to create conditions providing the concentration of polarization at a single node of the chain (up to the unavoidable experimental errors) [17]. The dynamic of quantum correlations in this model was first studied in [18]. Moreover, it was shown recently [19] that such chains are convenient for study of the dependence of the discord on the representation basis of the density matrix describing the state of a quantum system. Thus, the quantum discord calculated for the interacting nuclear spins differs from that between the fermions arising after the Jordan-Wigner transformation [20] of the density matrix operator [19, 21]. It seemed out that the quantum discord between fermions may exhibit very interesting properties [19] which have not been observed in the discord between nuclear spins. The most important property is the stationarity of the pairwise 
discord in a fermion cluster with the above initial state of a spin- $1 / 2$ chain. Besides, if we polarize the proper initial node, then the quantum discord will be the same for any fermion pair in the selected fermion cluster. Apparently, this fact is important for implementation of the fermion registers in the quantum devices because all fermion-nodes are equivalent from the quantum correlation point of view.

The existence of such clusters motivates the study of their stability with respect to both the experimental errors in creation of the single-node polarization and the noise effects. We note that the stability of the spin dynamics in the presence of different types of noise is an attractive problem, because noise is unavoidable in any quantum process. In particular, the fidelity of the perfect (in the absence of noises) state transfer under noise-perturbations of the coupling constants in the Hamiltonian was considered in refs. 222, 23, 24, 25, 26] for two chains: a completely engineered chain and a chain with remote end-nodes. In both cases, the important result is that the noise reduces the fidelity without changing the state transfer time.

In this paper we study the stability of the discord distribution relative to perturbations of the initial state in a homogeneous spin chain (i.e. the coupling constants in the Hamiltonian are assumed to be stable). We show that the stationarity of the quantum discord in the system with the single initially polarized node may not be destroyed by the additional low polarizations of the neighboring nodes which unavoidably appear in the experiment. This perturbation just leads to the deformation of the pairwise quantum discord and may eventually destroy the clusters of fermions with the equal pairwise discord. The threshold value of the low-polarization is found. We also consider the deformation of the stationary discord distribution caused by the noise-polarization appearing in all nodes of the spin chain. It is remarkable that the discord stationarity is not disturbed in both cases.

The paper is organized as follows. The Jordan-Wigner transformation of the $X Y$-Hamiltonian with nearest neighbor interactions is briefly discussed in Sec.2. The stability of the pairwise discord stationarity in the Jordan-Wigner fermion system of a spin-1/2 chain with single initially polarized node under perturbations of the initial state is demonstrated in Secs, 3 and 4 with the numerical simulations of the spin dynamics of a 17 -node chain. First, in Sec 3 , the parasitic polarization of two neighboring nodes (with respect to the selected inner polarized node) is considered. Then, in Sec4, the noise polarization of all nodes is taken into account using the perturbation method. Deformations of the fermion clusters with the equal pairwise discord under the above perturbations are also considered in Secs 3 and 4. The basic results are discussed in Sec.5. A formula for calculating the discord in the $X$-type density matrix [27] is represented in the Appendix, Sec 6. 


\section{Jordan-Wigner transformation of $X Y$-Hamiltonian with the nearest-neighbor interaction.}

Hereafter we study quantum correlations in the one-dimensional open spin-1/2 chain of $N$ nodes governed by the XY Hamiltonian with the nearest neighbor interactions,

$$
H=\omega_{0} \sum_{i=1}^{N} I_{i z}+D \sum_{i=1}^{N-1}\left(I_{i x} I_{(i+1) x}+I_{i y} I_{(i+1) y}\right),
$$

where $\omega_{0}$ is the Larmor frequency in the external magnetic field, $D$ is the spin-spin coupling constant between the nearest neighbors, and $I_{i \alpha}(i=1, \ldots, N, \alpha=x, y, z)$ is the $i$ th spin projection on the $\alpha$-axis.

Following refs. [18, 19, 21], we diagonalize Hamiltonian (1) using the Jordan-Wigner transformation method [20]

$$
H=\sum_{k} \varepsilon_{k} \beta_{k}^{+} \beta_{k}-\frac{1}{2} N \omega_{0}, \quad \varepsilon_{k}=D \cos (k)+\omega_{0},
$$

where the fermion operators $\beta_{j}$ are determined in terms of other fermion operators $c_{j}$ by means of the Fourier transformation

$$
\beta_{k}=\sum_{j=1}^{N} g_{k}(j) c_{j}
$$

and the fermion operators $c_{j}$ are determined as [20]

$$
c_{j}=(-2)^{j-1} I_{1 z} I_{2 z} \ldots I_{(j-1) z} I_{j}^{-} .
$$

Here,

$$
g_{k}(j)=\left(\frac{2}{N+1}\right)^{1 / 2} \sin (k j), \quad k=\frac{\pi n}{N+1}, \quad n=1,2, \ldots, N .
$$

We can readily express the projection operators $I_{j z}$ in terms of the fermion operators $c_{j}$ as

$$
I_{j z}=c_{j}^{+} c_{j}-\frac{1}{2}, \quad \forall j .
$$

Hereafter, diagonal representation (2) of the $X Y$ Hamiltonian will be used to describe the dynamics of the density matrix associated with the spin-1/2 chain.

\section{Initial state with three polarized nodes}

The dynamics of the Jordan-Wigner fermions associated with the spin- $1 / 2$ chain with single initially polarized node $j_{0}$ has been studied in refs.[18, 19, 21]. The stationarity of the pairwise 
discord in such systems is demonstrated therein. Also the fermion clusters with the equal pairwise discord are revealed in those references.

Now we consider the initial state with an inner initially polarized node $j_{0}$ (i.e., $1<j_{0}<N$ ) and assume the parasitic low polarization of two neighboring nodes, i.e. the initial density matrix reads

$$
\begin{aligned}
& \rho_{0}=\frac{e^{\sum_{k=j_{0}-1}^{j_{0}+1} b_{k} I_{k, z}}}{Z}=\frac{1}{2^{N}} \prod_{k=j_{0}-1}^{j_{0}+1}\left(1+2 I_{k z} \tanh \frac{b_{k}}{2}\right), \\
& Z=\operatorname{Tr}\left(\prod_{k=j_{0}-1}^{j_{0}+1} e^{b_{k} I_{k z}}\right)=2^{N} \prod_{k=j_{0}-1}^{j_{0}+1} \cosh \frac{b_{k}}{2}
\end{aligned}
$$

where $b_{j}=\frac{\hbar \omega_{j 0}}{k T}, \hbar$ is the Plank constant, $k$ is the Boltzmann constant, and $T$ is the temperature of the system.

The motivation for considering this initial state is mentioned in the introduction. Namely, an experimental scheme may not provide the ideal single-node polarization. So, two neighboring nodes $j_{0} \pm 1$ get some polarization as well provided that $j_{0}$ is the inner node, i.e. $1<j_{0}<N$. This polarization might be referred to as the parasitic one. As was shown in [19, 21], a fermion cluster with equal pairwise discords (which is of our interest in this paper) may be obtained if the polarized node $j_{0}$ is an inner one. This case is related to the density matrix (17) and is considered below.

The evolution of the initial density matrix (7) in virtue of the fermion representation of the Hamiltonian (2) reads

$$
\rho(t)=e^{-i t \sum_{k} \varepsilon_{k} \beta_{k}^{+} \beta_{k}} \rho_{0} e^{i t \sum_{k} \varepsilon_{k} \beta_{k}^{+} \beta_{k}}
$$

Using the identity

$$
e^{-i \varphi \beta_{k}^{+} \beta_{k}} \beta_{k}^{+} e^{i \varphi \beta_{k}^{+} \beta_{k}}=e^{-i \varphi} \beta_{k}^{+}, \quad \forall \varphi,
$$

we transfer density matrix (86) to the following form [18]

$$
\begin{aligned}
& \rho(t)=\frac{1}{2^{N}} \prod_{j=j_{0}-1}^{j_{0}+1}\left(1-\tanh \frac{b_{j}}{2}+2 \tanh \frac{b_{j}}{2} \sum_{k, k^{\prime}} e^{-i t\left(\varepsilon_{k}-\varepsilon_{k^{\prime}}\right)} g_{k}(j) g_{k^{\prime}}(j) \beta_{k}^{+} \beta_{k^{\prime}}\right)= \\
& A_{0}^{j_{0}}+\sum_{k, k^{\prime}} A_{k k^{\prime}}^{j_{0}} e^{-i t\left(\varepsilon_{k}-\varepsilon_{k^{\prime}}\right)} \beta_{k}^{+} \beta_{k^{\prime}}+\sum_{k, k^{\prime}, q, q^{\prime}} A_{k q k^{\prime} q^{\prime}}^{j_{0}} e^{-i t\left(\varepsilon_{k}+\varepsilon_{q}-\varepsilon_{k^{\prime}}-\varepsilon_{q^{\prime}}\right)} \beta_{k}^{+} \beta_{k^{\prime}} \beta_{q}^{+} \beta_{q^{\prime}}+ \\
& \sum_{k, k^{\prime}, q, q^{\prime}, l, l^{\prime}} A_{k q l k^{\prime} q^{\prime} l^{\prime}}^{j_{0}} e^{-i t\left(\varepsilon_{k}+\varepsilon_{q}+\varepsilon_{l}-\varepsilon_{k^{\prime}}-\varepsilon_{q^{\prime}}-\varepsilon_{l^{\prime}}\right)} \beta_{k}^{+} \beta_{k^{\prime}} \beta_{q}^{+} \beta_{q^{\prime}} \beta_{l}^{+} \beta_{l^{\prime}}
\end{aligned}
$$


where

$$
\begin{aligned}
& A_{0}^{j_{0}}=\frac{1}{2^{N}} \prod_{j=j_{0}-1}^{j_{0}+1}\left(1-\tanh \frac{b_{j}}{2}\right) \\
& A_{k k^{\prime}}^{j_{0}}=\frac{1}{2^{N-1}}( \\
& \left(1-\tanh \frac{b_{j_{0}}}{2}\right)\left(1-\tanh \frac{b_{j_{0}+1}}{2}\right) \tanh \frac{b_{j_{0}-1}}{2} g_{k}\left(j_{0}-1\right) g_{k^{\prime}}\left(j_{0}-1\right)+ \\
& \left(1-\tanh \frac{b_{j_{0}-1}}{2}\right)\left(1-\tanh \frac{b_{j_{0}+1}}{2}\right) \tanh \frac{b_{j_{0}}}{2} g_{k}\left(j_{0}\right) g_{k^{\prime}}\left(j_{0}\right)+ \\
& \left.\left(1-\tanh \frac{b_{j_{0}-1}}{2}\right)\left(1-\tanh \frac{b_{j_{0}}}{2}\right) \tanh \frac{b_{j_{0}+1}}{2} g_{k}\left(j_{0}+1\right) g_{k^{\prime}}\left(j_{0}+1\right)\right) \\
& A_{k q k^{\prime} q^{\prime}}^{j_{0}}=\frac{1}{2^{N-2}}\left(1-\tanh \frac{b_{j_{0}-1}}{2}\right) \tanh \frac{b_{j_{0}}}{2} \tanh \frac{b_{j_{0}+1}}{2} g_{k}\left(j_{0}\right) g_{k^{\prime}}\left(j_{0}\right) g_{q}\left(j_{0}+1\right) g_{q^{\prime}}\left(j_{0}+1\right)+ \\
& \left(1-\tanh \frac{b_{j_{0}}}{2}\right) \tanh \frac{b_{j_{0}-1}}{2} \tanh \frac{b_{j_{0}+1}}{2} g_{k}\left(j_{0}-1\right) g_{k^{\prime}}\left(j_{0}-1\right) g_{q}\left(j_{0}+1\right) g_{q^{\prime}}\left(j_{0}+1\right)+ \\
& \left.\left(1-\tanh \frac{b_{j_{0}+1}}{2}\right) \tanh \frac{b_{j_{0}-1}}{2} \tanh \frac{b_{j_{0}}}{2} g_{k}\left(j_{0}-1\right) g_{k^{\prime}}\left(j_{0}-1\right) g_{q}\left(j_{0}\right) g_{q^{\prime}}\left(j_{0}\right)\right) \\
& A_{k q l k^{\prime} q^{\prime} l^{\prime}}^{j_{0}}=\frac{b_{j_{0}-1}}{2} \tanh \frac{b_{j_{0}}}{2} \tanh \frac{b_{j_{0}+1}}{2} g_{k}\left(j_{0}-1\right) g_{k^{\prime}}\left(j_{0}-1\right) g_{q}\left(j_{0}\right) g_{q^{\prime}}\left(j_{0}\right) g_{l}\left(j_{0}+1\right) g_{l^{\prime}}\left(j_{0}+1\right) \\
& \left.\frac{1}{2^{N-3}} \tanh \frac{b}{2}\right)
\end{aligned}
$$

Finally, using the fermion anti-commutation relations [28] and relations among the coefficients $A_{k q k^{\prime} q^{\prime}}^{j_{0}}$ and $A_{k q l k^{\prime} q^{\prime} l^{\prime}}^{j_{0}}$

$$
\sum_{q=1}^{N} A_{k q q k^{\prime}}^{j 0}=\sum_{q=1}^{N} A_{k l q k^{\prime} q l^{\prime}}^{j 0}=\sum_{q=1}^{N} A_{k q l q k^{\prime} l^{\prime}}^{j 0}=\sum_{q=1}^{N} A_{k l q q k^{\prime} l^{\prime}}^{j 0}=0
$$

which follow from the orthogonality relationship

$$
\sum_{k=1}^{N} g_{k}(j) g_{k}(l)=\delta_{k l}
$$


we easily transform eq.(10) to the following canonic form:

$$
\begin{aligned}
& \rho(t)= \\
& \frac{1}{2^{N}} \prod_{j=j_{0}-1}^{j_{0}+1}\left(1-\tanh \frac{b_{j}}{2}\right)+\sum_{k, k^{\prime}} A_{k k^{\prime}}^{j_{0}} e^{-i t\left(\varepsilon_{k}-\varepsilon_{k^{\prime}}\right)} \beta_{k}^{+} \beta_{k^{\prime}}- \\
& \sum_{\substack{k, k^{\prime}, q, q^{\prime} \\
k \neq q, k^{\prime} \neq q^{\prime}}}^{j_{k q k^{\prime} q^{\prime}}} e^{-i t\left(\varepsilon_{k}+\varepsilon_{q}-\varepsilon_{k^{\prime}}-\varepsilon_{q^{\prime}}\right)} \beta_{k}^{+} \beta_{q}^{+} \beta_{k^{\prime}} \beta_{q^{\prime}}- \\
& \sum_{\substack{k, k^{\prime}, q, q^{\prime}, l, l^{\prime} \\
k \neq q \neq l, k^{\prime} \neq q^{\prime} \neq l^{\prime}}}^{j_{0}} A_{k q l k^{\prime} q^{\prime} l^{\prime}}^{j_{0}}
\end{aligned}
$$

\subsection{Reduced density matrix}

Studing quantum correlations we consider only the pairwise discord. First, in calculation of the discord between the $n$th and $m$ th fermions, we have to reduce density matrix (14) with respect to all nodes except for the $n$th and $m$ th ones resulting to the marginal density matrix of the following form:

$$
\rho_{n m}^{j_{0}}(t)=B_{n m}^{j_{0}}+\sum_{k, k^{\prime}=n, m} B_{n m k k^{\prime}}^{j_{0}} e^{-i t\left(\varepsilon_{k}-\varepsilon_{k^{\prime}}\right)} \beta_{k}^{+} \beta_{k^{\prime}}+C_{n m}^{j_{0}} \beta_{n}^{+} \beta_{m}^{+} \beta_{m} \beta_{n}
$$


where all coefficients do not depend on the time $t$ :

$$
\begin{aligned}
& B_{n m}^{j_{0}}=\frac{1}{4} \prod_{j=j_{0}-1}^{j_{0}+1}\left(1-\tanh \frac{\beta_{j}}{2}\right)+2^{N-3} \sum_{k \neq n, m} A_{k k}^{j_{0}}+2^{N-4} \sum_{\substack{k, q \\
k \neq q ; k, q \neq n, m}}\left(-A_{k q k q}^{j_{0}}+A_{k q q k}^{j_{0}}\right)- \\
& 2^{N-5} \sum_{\substack{k, q, l \neq n, m \\
k \neq q \neq l}}\left(-A_{k q l k q l}^{j_{0}}+A_{k q l k l q}^{j_{0}}+A_{k q l q k l}^{j_{0}}-A_{k q l l k q}^{j_{0}}-A_{k q l q l k}^{j_{0}}+A_{k q l l q k}^{j_{0}}\right) \\
& B_{n m k k^{\prime}}^{j_{0}}=2^{N-2} A_{k k^{\prime}}^{j_{0}}+2^{N-3} \sum_{q, q \neq k, k^{\prime}}\left(-A_{k q k^{\prime} q}^{j_{0}}+A_{k q q k^{\prime}}^{j_{0}}+A_{q k k^{\prime} q}^{j_{0}}-A_{q k q k^{\prime}}^{j_{0}}\right)- \\
& 2^{N-4} \sum_{\substack{q, l \\
q \neq l \neq k \neq k^{\prime}}}\left(-A_{k q l k^{\prime} q l}^{j_{0}}+A_{k q l k^{\prime} l q}^{j_{0}}+A_{k q l q k^{\prime} l}^{j_{0}}-A_{k q l l k^{\prime} q}^{j_{0}}-A_{k q l q l k^{\prime}}^{j_{0}}+A_{k q l l q k^{\prime}}^{j_{0}}+A_{q k l k^{\prime} q l}^{j_{0}}-\right. \\
& A_{q k l k^{\prime} l q}^{j_{0}}-A_{q k l q k^{\prime} l}^{j_{0}}+A_{q k l l k^{\prime} q}^{j_{0}}+A_{q k l q l k^{\prime}}^{j_{0}}-A_{q k l l q k^{\prime}}^{j_{0}}- \\
& \left.A_{q l k k^{\prime} q l}^{j_{0}}+A_{q l k k^{\prime} l q}^{j_{0}}+A_{q l k q k^{\prime} l}^{j_{0}}-A_{q l k l k^{\prime} q}^{j_{0}}-A_{q l k q l k^{\prime}}^{j_{0}}+A_{q l k l q k^{\prime}}^{j_{0}}\right) \\
& C_{n m}^{j_{0}}=2^{N-2}\left(A_{n m m n}^{j_{0}}-A_{n m n m}^{j_{0}}-A_{m n m n}^{j_{0}}+A_{m n n m}^{j_{0}}\right)- \\
& 2^{N-3} \sum_{k \neq n, m}\left(A_{k n m k m n}^{j_{0}}-A_{k n m k n m}^{j_{0}}-A_{k m n k m n}^{j_{0}}+A_{k m n k n m}^{j_{0}}-\right. \\
& A_{k n m m k n}^{j_{0}}+A_{k n m n k m}^{j_{0}}+A_{k m n m k n}^{j_{0}}-A_{k m n n k m}^{j_{0}}+ \\
& A_{k n m m n k}^{j_{0}}-A_{k n m n m k}^{j_{0}}-A_{k m n m n k}^{j_{0}}+A_{k m n n m k}^{j_{0}}-A_{n k m k m n}^{j_{0}}+A_{n k m k n m}^{j_{0}}+A_{m k n k m n}^{j_{0}}-A_{m k n k n m}^{j_{0}}+ \\
& A_{n k m m k n}^{j_{0}}-A_{n k m n k m}^{j_{0}}-A_{m k n m k n}^{j_{0}}+A_{m k n n k m}^{j_{0}}-A_{n k m m n k}^{j_{0}}+A_{n k m n m k}^{j_{0}}+A_{m k n m n k}^{j_{0}}-A_{m k n n m k}^{j_{0}}+ \\
& A_{n m k k m n}^{j_{0}}-A_{n m k k n m}^{j_{0}}-A_{m n k k m n}^{j_{0}}+A_{m n k k n m}^{j_{0}}-A_{n m k m k n}^{j_{0}}+A_{n m k n k m}^{j_{0}}+A_{m n k m k n}^{j_{0}}-A_{m n k n k m}^{j_{0}}+ \\
& \left.A_{n m k m n k}^{j_{0}}-A_{n m k n m k}^{j_{0}}-A_{m n k m n k}^{j_{0}}+A_{m n k n m k}^{j_{0}}\right)
\end{aligned}
$$

Next, using the basis

$$
|00\rangle,|01\rangle,|10\rangle,|11\rangle,
$$

we represent the marginal density matrix operator (15) in the following matrix form:

$$
\rho_{n m}^{j_{0}}(t)=\left(\begin{array}{cccc}
B_{n m}^{j_{0}} & 0 & 0 & 0 \\
0 & B_{n m}^{j_{0}}+B_{n m n n}^{j_{0}} & B_{n m n m}^{j_{0}} e^{-i t\left(\varepsilon_{n}-\varepsilon_{m}\right)} & 0 \\
0 & B_{n m m n}^{j_{0}} e^{i t\left(\varepsilon_{n}-\varepsilon_{m}\right)} & B_{n m}^{j_{0}}+B_{n m m m}^{j_{0}} & 0 \\
0 & 0 & 0 & B_{n m}^{j_{0}}+B_{n m m}^{j_{0}}+B_{n m n n}^{j_{0}}+C_{n m}^{j_{0}}
\end{array}\right) .
$$

\subsubsection{Stationarity of the pairwise discord}

Formula (18) shows that the diagonal elements of marginal matrix (18) do not depend on the time $t$. The $t$-dependence appears only in non-diagonal elements. However, the pairwise discord for the $X$-matrix depends on the absolute value $\left|B_{n m n m}^{j_{0}}\right|$ of the non-diagonal element (see the Appendix, Sec 6) and, consequently, does not depend on $t$. This means that the perturbations considered in this section do not destroy the stationarity of the discord. However, the distribution of the discord becomes deformed which, eventually, may destroy the fermion clusters with equal pairwise discords. Deformations caused by the parasitic polarizations of $\left(j_{0} \pm 1\right)$ th nodes are studied numerically in the following subsection. 


\section{$3.2 \quad$ Numerical simulations}

We represent numerical simulations for a particular case of $N=17$ node spin chain and assume that the polarization is initially concentrated at such a node $j_{0}$ that the fermion clusters with equal pairwise discord may be selected from the whole system of 17 fermions [21]. The interest in this case appears because such clusters might be promising in the QIP-devices as candidates for large quantum registers.

In accordance with [19, 21], such a cluster $C l$ appears in an odd-node spin chain in two cases. First, if $j_{0}$ is the middle node $\left(j_{0}=9\right.$ in our case), then the cluster $C l$ is formed by the odd-fermions. Second, if $N=5+6 i(i=1,2, \ldots)$ and $j_{0}=2(i+1)$ (in our case $N=17, i=2$, so that $\left.j_{0}=6\right)$, then the cluster $C l$ is formed by the all fermions except each third one. In both cases, the pairwise discord $Q_{n m}$ between the $n$th and $m$ th node is

$$
Q_{n m}= \begin{cases}Q_{0}=\text { const, } & n \in C l, m \in C l \\ 0, & n \notin C l \text { and } / \text { or } m \notin C l .\end{cases}
$$

In other words, the discord $Q_{n m}$ is zero if at least one of the subscripts $n$ or $m$ is not in the set $\mathrm{Cl}$.

Thus, we consider two clusters corresponding to two cases of the initially polarized node $j_{0}$ :

1. $j_{0}=6$, the cluster of fermions with equal pairwise discords is formed by all fermions except each third one:

$$
C l=\{1,2,4,5,7,8,10,11,13,14,16,17\} .
$$

2. $j_{0}=9$, the odd fermions form the cluster with equal pairwise discords:

$$
C l=\{1,3,5, \ldots, 17\} .
$$

We characterize the polarization by the inverse temperatures

$$
\begin{aligned}
& b_{j_{0}+1}=b_{j_{0}-1}=b, \quad 0 \leq b \leq b_{j_{0}}, \\
& b_{j}=0, \quad j \neq j_{0}, j_{0} \pm 1 .
\end{aligned}
$$

In practice, $b$ must be such that $\tanh \frac{b_{j_{0} \pm 1}}{2}$ is several times less then $\tanh \frac{b_{j_{0}}}{2}$. Below we take $b_{j_{0}}=10$ (the low temperature limit).

The presence of the parasitic polarization leads to a deformation of the ideal (when $b_{j_{0} \pm 1}=0$ ) discord distribution shown in Fig 2a a. As a result, some spread of discord appears in the cluster $C l$. Besides, the zero-valued discords at $b=0$ become non-zero for $b>0$.

To characterize both these effects of parasitic polarization, we introduce the following functions

$$
\begin{aligned}
& C l_{\text {max }}(b)=\max _{(n, m) \in C l} Q_{n m}(b), \quad C l_{\text {min }}(b)=\min _{(n, m) \in C l} Q_{n m}(b), \\
& Z_{\text {max }}(b)=\max _{(n, m) \notin C l} Q_{n m}(b), \quad Z_{\text {min }}(b)=\min _{(n, m) \notin C l} Q_{n m}(b) .
\end{aligned}
$$



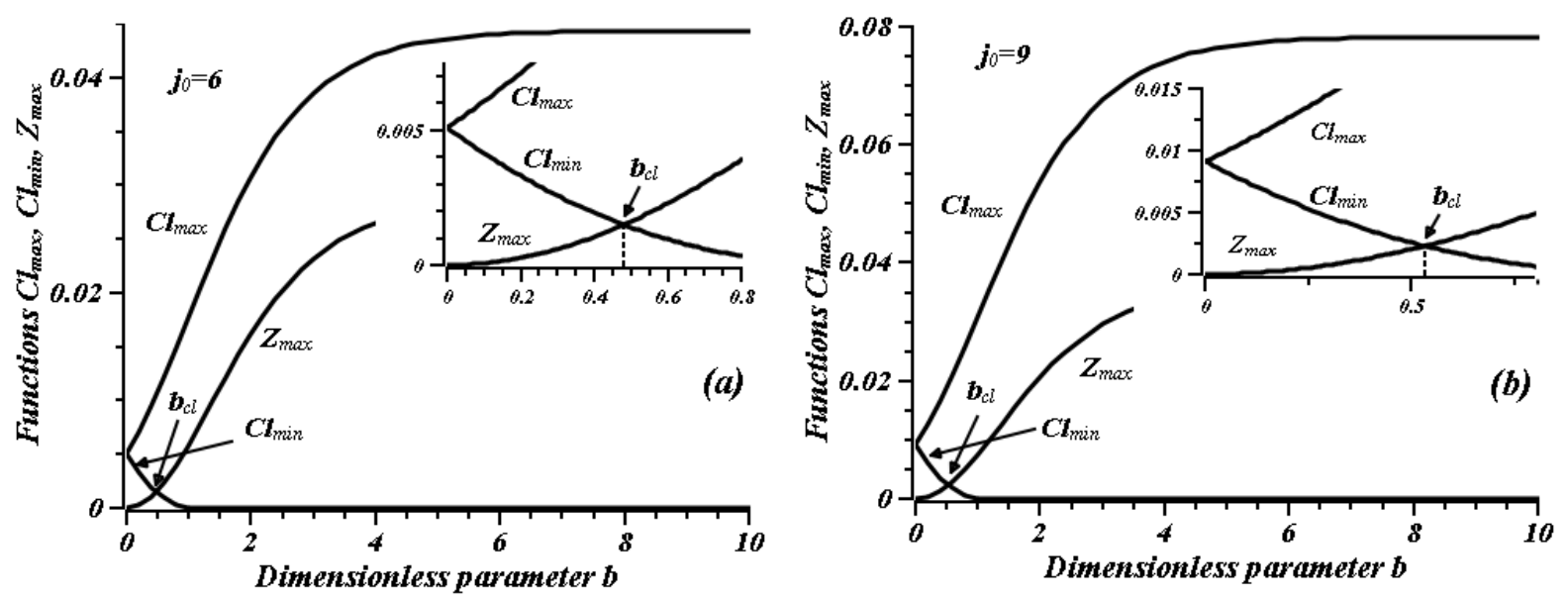

Figure 1: Deformations of fermion cluster in the system of $N=17$ fermions. The graphs of the functions $C l_{\max }(b), C l_{\min }(b)$ and $Z_{\max }(b)$ are shown. The intersection point $b_{c l}$ of the graphs $C l_{\min }(b)$ and $Z_{\max }(b)$ can be considered as the critical value of $b$ such that the cluster $C l$ of the correlated fermions does not exist if $b>b_{c l}$. The insets show the graphs of $C l_{\max }(b), C l_{\min }(b)$ and $Z_{\text {max }}(b)$ for small values of $b$. (a) The initially polarized node $j_{0}=6$, the critical value of the parameter $b$ is $b_{c l}=0.480$. (b) The initially polarized node $j_{0}=9$, the critical value $b_{c l}=0.533$.

Here, the notation $(n, m) \notin C l$ means $n \notin C l$ and/or $m \notin C l$. Functions $C l_{\text {max }}$ and $C l_{\text {min }}$ characterize the spread of the pairwise discord in clusters (20) and (21), while $Z_{\max }$ and $Z_{\min }$ characterize the spread of the "parasitic" discord that was zero in the unperturbed case, i.e.

$$
\begin{aligned}
& C l_{\text {min }}(b) \leq Q_{n m} \leq C l_{\text {max }}(b), \quad n \in C l, m \in C l, \\
& Z_{\text {min }}(b) \leq Q_{n m} \leq Z_{\max }(b), \quad n \notin C l \text { and/or } m \notin C l .
\end{aligned}
$$

The graphs of the functions $C l_{\max }(b), C l_{\min }(b)$ and $Z_{\max }(b)$ for $j_{0}=6$ and $j_{0}=9$ are shown in Fig [1 a a Fig 1b respectively. The function $Z_{\text {min }}(b)$ (although it is non-zero for $b>0$ ) is not shown because it is not important in this section. But it will be used in Sec4.1 to characterize the noise effects.

A natural question is about the critical value of the parameter $b$ (characterizing the value of the "parasitic" polarization) that still does not completely destroy the cluster $C l$. We considered the value of $b$ corresponding to the intersection point of $C l_{\min }(b)$ and $Z_{\max }(b)$ as the critical value $b_{c l}$, so that the cluster $C l$ does not exist if $b>b_{c l}$. The critical value is $b_{c l}=0.480$ for $j_{0}=6$ and $b_{c l}=0.533$ for $j_{0}=9$, as shown in Fig.1a,b.

An example of the discord distribution for $j_{0}=6$ and $b=b_{c l}=0.48$ (at the threshold value) is shown in Fig,2b. We see that this distribution significantly differs from the unperturbed case $b=0$ shown in Fig 2 a. However, we emphasize ones again, the parasitic polarization does not lead to the evolution of the discord in the considered fermion system, i.e., the stationarity of the pairwise discord is not destroyed. 

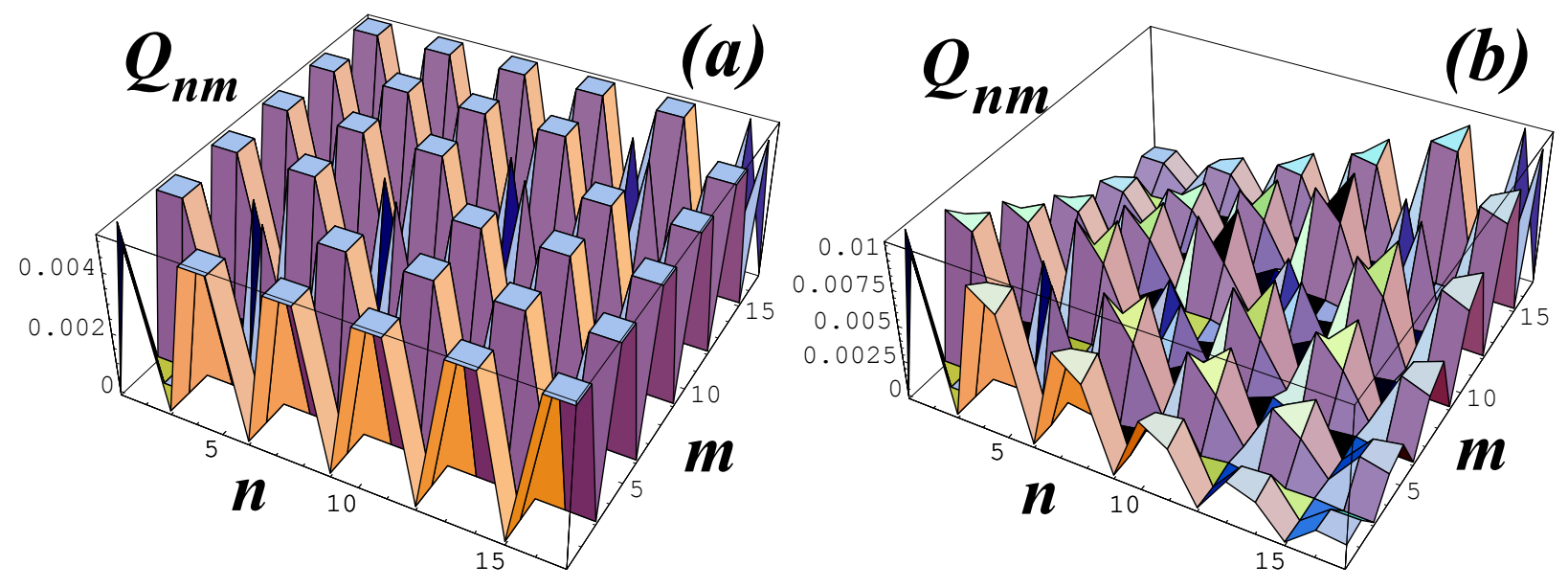

Figure 2: Distribution of the discord $Q_{n m}$ in the system of $N=17$ fermions with the initially polarized node $j_{0}=6$. Here and in Fig. 4 we put $Q_{n n} \equiv 0$ following ref.[21]. (a) Discord $Q_{n m}$ in the fermion system without parasitic polarization, $b=0 ;(b)$ Discord $Q_{n m}$ in the fermion system with the critical parasitic polarization of the 5 th and 7 th nodes, $b=b_{c l}=0.480$.

\section{Noise effect on the pairwise discord distribution}

In this section, we assume that there is no parasitic polarization considered in Sec 3 (i.e. $b_{j_{0} \pm 1}=$ 0 ), but there is noise-polarization of all nodes. The initial density matrix (7) must be replaced with the following one:

$$
\rho_{0}=\frac{1}{2^{N}}\left(1+I_{j_{0} z}\left(2 \tanh \frac{b_{j_{0}}}{2}+\epsilon \tilde{b}_{j_{0}}\right)\right) \prod_{\substack{k=1 \\ k \neq j_{0}}}^{N}\left(1+I_{k z} \epsilon \tilde{b}_{k}\right), \quad Z=2^{N} \cosh \frac{b_{j_{0}}}{2}
$$

where $\epsilon$ is the double noise-polarization amplitude in a spin- $1 / 2$ chain, $\epsilon \ll 1$, and $\tilde{b}_{j}$ are the random numbers inside of the interval $-\frac{1}{2} \leq \tilde{b}_{j} \leq \frac{1}{2}$. Using eqs.(3), ([6]) we transform the initial density matrix to the following form [18]

$$
\rho_{0}=\frac{1}{2^{N}}\left(a_{0}^{j_{0}}+\sum_{k, k^{\prime}} a_{k k^{\prime}}^{j_{0}} \beta_{k}^{+} \beta_{k^{\prime}}\right) \prod_{\substack{j=1 \\ j \neq j_{0}}}^{N}\left(a_{0}^{j}+\sum_{k, k^{\prime}} a_{k k^{\prime}}^{j} \beta_{k}^{+} \beta_{k^{\prime}}\right)
$$

where

$$
\begin{aligned}
& a_{0}^{j}=\left\{\begin{array}{ll}
1-\tanh \frac{b_{j_{0}}}{2}-\frac{\epsilon \tilde{\epsilon}_{j_{0}}}{2}, & j=j_{0} \\
1-\frac{\epsilon \tilde{b}_{j}}{2}, & j \neq j_{0}
\end{array},\right. \\
& a_{k k^{\prime}}^{j}=\left\{\begin{array}{ll}
\left(2 \tanh \frac{b_{j_{0}}}{2}+\epsilon \tilde{b}_{j_{0}}\right) g_{k}\left(j_{0}\right) g_{k^{\prime}}\left(j_{0}\right), & j=j_{0} \\
\epsilon \tilde{b}_{j} g_{k}(j) g_{k^{\prime}}(j), & j \neq j_{0}
\end{array} .\right.
\end{aligned}
$$

The quantities $a_{k k^{\prime}}^{j}, j \neq j_{0}$, are proportional to $\epsilon$ and thus they are considered as small parameters hereafter. 
Now we assume that the noise effect can be studied by the perturbation method for small $\epsilon$. Thus, we expand the initial density matrix in the $a_{k k^{\prime}}^{j}, j \neq j_{0}$. We consider two density matrices cutting the series and keeping the terms up to the order, respectively, $a_{k k^{\prime}}^{j}$ and $\left(a_{k k^{\prime}}^{j}\right)^{2}$. Taking into account the normalization condition (trace of density matrix equals unit) we write these matrices as follows:

$$
\begin{aligned}
& \rho_{0 i}=\frac{\tilde{\rho}_{0 i}}{Z_{i}}, Z_{i}=\operatorname{Tr} \tilde{\rho}_{0 i}, \quad i=1,2 \\
& \tilde{\rho}_{01}=\frac{1}{2^{N}}\left(a_{0}^{j_{0}}+\sum_{k, k^{\prime}} a_{k k^{\prime}}^{j_{0}} \beta_{k}^{+} \beta_{k^{\prime}}\right)\left(\prod_{\substack{l=1 \\
l \neq j_{0}}}^{N} a_{0}^{l}+\sum_{\substack{n=1 \\
n \neq j_{0}}}^{N}\left(\prod_{\substack{m=1 \\
m \neq j_{0} \neq n}}^{N} a_{0}^{m}\right) \sum_{k, k^{\prime}} a_{k k^{\prime}}^{n} \beta_{k}^{+} \beta_{k^{\prime}}\right), \\
& \tilde{\rho}_{02}=\frac{1}{2^{N}}\left(a_{0}^{j_{0}}+\sum_{k, k^{\prime}} a_{k k^{\prime}}^{j_{0}} \beta_{k}^{+} \beta_{k^{\prime}}\right) \times \\
& \left(\prod_{\substack{l=1 \\
l \neq j_{0}}}^{N} a_{0}^{l}+\sum_{\substack{n=1 \\
n \neq j_{0}}}^{N}\left(\prod_{\substack{m=1 \\
m \neq j_{0} \neq n}}^{N} a_{0}^{m}\right) \sum_{k, k^{\prime}} a_{k k^{\prime}}^{n} \beta_{k}^{+} \beta_{k^{\prime}}+\sum_{\substack{n, n^{\prime}=1 \\
n^{\prime} \neq n \neq j_{0}}}^{N}\left(\prod_{\substack{m=1 \\
m \neq j_{0} \neq n \neq n^{\prime}}}^{N} a_{0}^{m}\right) \sum_{\substack{k, k^{\prime}, q, q^{\prime} \\
k k^{\prime}}} a_{\substack{q^{\prime} \\
n^{\prime}}}^{n} \beta_{k}^{+} \beta_{k^{\prime}} \beta_{q}^{+} \beta_{q^{\prime}}\right) .
\end{aligned}
$$

The evolution of these matrices $e^{-i t H} \rho_{0 i} e^{i t H}$, taking into account the fermion representation of the Hamiltonian (given by formula (2) ) and relation (9), reads:

$$
\begin{aligned}
& \rho_{i}(t)=\frac{\tilde{\rho}_{i}(t)}{Z_{i}}, \\
& \tilde{\rho}_{1}(t)=\frac{1}{2^{N}}\left(a_{0}^{j_{0}}+\sum_{k, k^{\prime}} a_{k k^{\prime}}^{j_{0}} e^{-i t\left(\varepsilon_{k}-\varepsilon_{k^{\prime}}\right)} \beta_{k}^{+} \beta_{k^{\prime}}\right) \times \\
& \left(\prod_{\substack{l=1 \\
l \neq j_{0}}}^{N} a_{0}^{l}+\sum_{\substack{n=1 \\
n \neq j_{0}}}^{N}\left(\prod_{\substack{m=1 \\
m \neq j_{0} \neq n}}^{N} a_{0}^{m}\right) \sum_{k, k^{\prime}} a_{k k^{\prime}}^{n} e^{-i t\left(\varepsilon_{k}-\varepsilon_{k^{\prime}}\right)} \beta_{k}^{+} \beta_{k^{\prime}}\right), \\
& \tilde{\rho}_{2}(t)=\frac{1}{2^{N}}\left(a_{0}^{j_{0}}+\sum_{k, k^{\prime}} a_{k k^{\prime}}^{j_{0}} e^{-i t\left(\varepsilon_{k}-\varepsilon_{k^{\prime}}\right)} \beta_{k}^{+} \beta_{k^{\prime}}\right) \times \\
& \left(\prod_{\substack{l=1 \\
l \neq j_{0}}}^{N} a_{0}^{l}+\sum_{\substack{n=1 \\
n \neq j_{0}}}^{N}\left(\prod_{\substack{m=1 \\
m \neq j_{0} \neq n}}^{N} a_{0}^{m}\right) \sum_{k, k^{\prime}} a_{k k^{\prime}}^{n} e^{-i t\left(\varepsilon_{k}-\varepsilon_{k^{\prime}}\right)} \beta_{k}^{+} \beta_{k^{\prime}}+\right. \\
& \left.\sum_{\substack{n, n^{\prime}=1 \\
n^{\prime} \neq n \neq j_{0}}}^{N}\left(\prod_{\substack{m=1 \\
m \neq j_{0} \neq n \neq n^{\prime}}}^{N} a_{0}^{m}\right) \sum_{k, k^{\prime}, q, q^{\prime}} a_{k k^{\prime}}^{n} a_{q q^{\prime}}^{n^{\prime}} e^{-i t\left(\varepsilon_{k}+\varepsilon_{q}-\varepsilon_{k^{\prime}}-\varepsilon_{q^{\prime}}\right)} \beta_{k}^{+} \beta_{k^{\prime}} \beta_{q}^{+} \beta_{q^{\prime}}\right) .
\end{aligned}
$$


Formulas (30) may be transformed to form (10) with

$$
\begin{aligned}
& A_{0}^{j_{0}}=\frac{1}{2^{N}} \prod_{l=1}^{N} a_{0}^{l} \\
& A_{k k^{\prime}}^{j_{0}}=\frac{1}{2^{N}}\left(a_{0}^{j_{0}} \sum_{\substack{n=1 \\
n \neq j_{0}}}^{N}\left(\prod_{\substack{m=1 \\
m \neq j_{0} \neq n}}^{N} a_{0}^{m}\right) a_{k k^{\prime}}^{n}+a_{k k^{\prime}}^{j_{0}} \prod_{\substack{l=1 \\
l \neq j_{0}}}^{N} a_{0}^{l}\right) \\
& A_{k q k^{\prime} q^{\prime}}^{j_{0}}=\frac{a_{k k^{\prime}}^{j_{0}}}{2^{N}} \sum_{\substack{n=1 \\
n \neq j_{0}}}^{N}\left(\prod_{\substack{m=1 \\
m \neq j_{0} \neq n}}^{N} a_{0}^{m}\right) a_{q q^{\prime}}^{n}, \\
& A_{k q l k^{\prime} q^{\prime} l^{\prime}}^{j_{0}}=0
\end{aligned}
$$

for the density matrix $\rho_{1}$, or

$$
\begin{aligned}
& A_{0}^{j_{0}}=\frac{1}{2^{N}} \prod_{l=1}^{N} a_{0}^{l}, \\
& A_{k k^{\prime}}^{j_{0}}=\frac{1}{2^{N}}\left(a_{0}^{j_{0}} \sum_{\substack{n=1 \\
n \neq j_{0}}}^{N}\left(\prod_{\substack{m=1 \\
m \neq j_{0} \neq n}}^{N} a_{0}^{m}\right) a_{k k^{\prime}}^{n}+a_{k k^{\prime}}^{j_{0}}\left(\prod_{\substack{l=1 \\
l \neq j_{0}}}^{N} a_{0}^{l}\right)\right) \\
& A_{k q k^{\prime} q^{\prime}}^{j_{0}}=\frac{1}{2^{N}}\left(a_{k k^{\prime}}^{j_{0}} \sum_{\substack{n=1 \\
n \neq j_{0}}}^{N}\left(\prod_{\substack{m=1 \\
m \neq j_{0} \neq n}}^{N} a_{0}^{m}\right) a_{q q^{\prime}}^{n}+a_{0}^{j_{0}} \sum_{\substack{n, n^{\prime}=1 \\
n^{\prime} \neq n \neq j_{0}}}^{N}\left(\prod_{\substack{m=1 \\
m \neq j_{0} \neq n \neq n^{\prime}}}^{N} a_{0}^{m}\right) a_{k k^{\prime}}^{n} a_{q q^{\prime}}^{n^{\prime}}\right) \\
& A_{k q l k^{\prime} q^{\prime} l^{\prime}}^{j_{0}}=\frac{a_{k k^{\prime}}^{j_{0}}}{2^{N}} \sum_{\substack{n, n^{\prime}=1 \\
n^{\prime} \neq n \neq j_{0}}}^{N}\left(\prod_{\substack{m=1 \\
m \neq j_{0} \neq n \neq n^{\prime}}}^{N} a_{0}^{m}\right) a_{q q^{\prime}}^{n} a_{l l^{\prime}}^{n^{\prime}}
\end{aligned}
$$

for the density matrix $\rho_{2}$. In this section, formulas (14-18) hold as well. The stationarity of the pairwise quantum discord between fermions follows from the structure of the marginal matrix $\rho_{n m}^{j_{0}}$ (18) and can be shown in a way similar to that proposed in Sec.3.

\subsection{Numerical simulations}

Similar to the numerical simulations of Sec 3.2, we perform the numerical simulations for the particular case of $N=17$ node spin chain with the initially polarized spins $j_{0}=6$

and $j_{0}=9$. For each fixed value of the small parameter $\epsilon$ in the interval $0 \leq \epsilon \leq 0.4$ $(\epsilon=0,0.1,0.2,0.3,0.4)$ we average the discord over $10^{2}$ realizations of the random set of the parameters $\tilde{b}_{j}, j=1, \ldots, 17$, characterizing the noise-polarization of the $j$ th node of the spin-1/2 chain. For the averaged discord, we use the same notation $Q_{n m}$ in this section. 

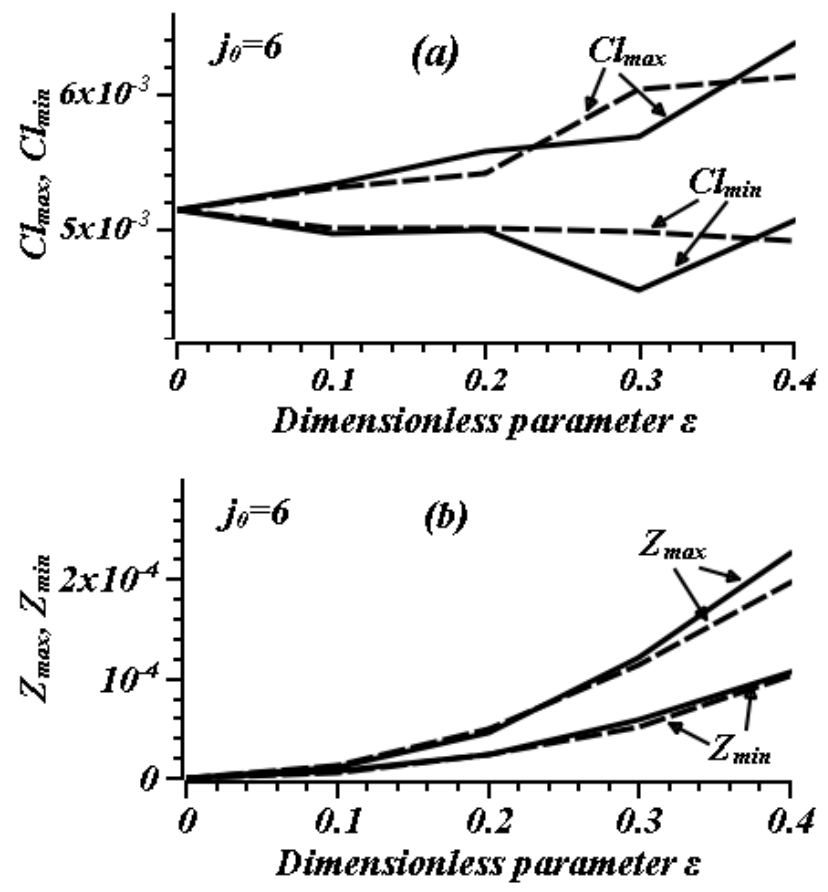
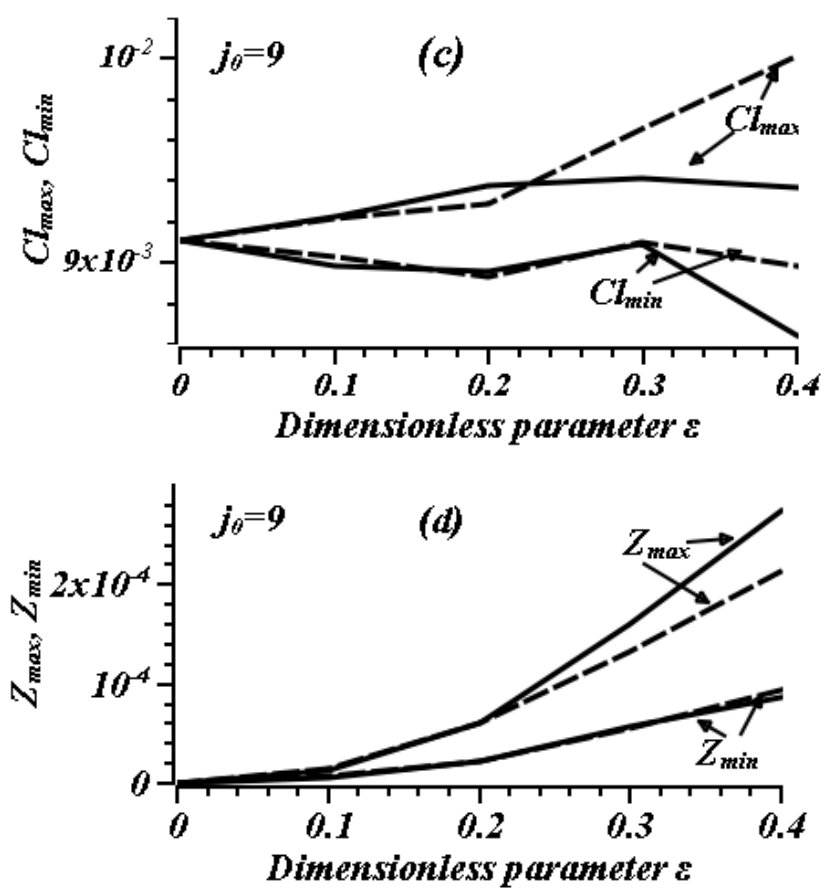

Figure 3: Deformations of the fermion cluster in the system of $N=17$ fermions with the noisepolarization of the initial state. The pairwise discord is averaged over $10^{2}$ realizations of the random choices of the parameters $\tilde{b}_{j}, j=1, \ldots, 17$, for each fixed value of the small parameter $\epsilon, \epsilon=0,0.1,0.2,0.3,0.4$. The cluster deformation by noise effects for the density matrix $\rho_{1}$ (solid lines) and $\rho_{2}$ (dashed lines) is characterized by the functions $C l_{\max }(\epsilon), C l_{\min }(\epsilon), Z_{\max }(\epsilon)$, $Z_{\min }(\epsilon)$. (a) The initially polarized node $j_{0}=6$, the functions $C l_{\max }(\epsilon)$ and $C l_{\min }(\epsilon)$. (b) The initially polarized node $j_{0}=6$, the functions $Z_{\max }(\epsilon)$ and $Z_{\min }(\epsilon) ;(c)$ The initially polarized node $j_{0}=9$, the functions $C l_{\max }(\epsilon)$ and $C l_{\min }(\epsilon) ;(d)$ The initially polarized node $j_{0}=9$, the functions $Z_{\max }(\epsilon)$ and $Z_{\min }(\epsilon)$.

Again, to characterize the deformation of the discord distribution caused by the noisepolarization, we use the functions $C l_{\max }(\epsilon), C l_{\min }(\epsilon)$ and $Z_{\max }(\epsilon), Z_{\min }(\epsilon)$ defined by formulas (231) and (24) in which we replace $b$ with $\epsilon$ to characterize the spread of the discord in the cluster $\mathrm{Cl}$ and the spread of the parasitic discord, which is zero in the absence of noise. Pairs of functions $C l_{\max }(\epsilon), C l_{\min }(\epsilon)$ and $Z_{\max }(\epsilon), Z_{\min }(\epsilon)$ are shown, respectively, in Figs $3 \mathrm{a}$ and $3 \mathrm{~b}$ for $j_{0}=6$ and in Figs $3 \mathrm{c}$ and $3 \mathrm{H}$ for $j_{0}=9$. We see that the difference between the discord distribution corresponding to the density matrices $\rho_{1}$ and $\rho_{2}$ is not significant inside the interval $0 \leq \epsilon \leq 0.4$, as is shown in Figs.3. More exactly, the curves $C l_{\max }$ corresponding to the maximal discord for the density matrices $\rho_{1}$ and $\rho_{2}$ are close to each other (see the upper solid and dashed lines in Figs $\sqrt[3]{3}$ and $3 \mathrm{k}$ ), as well as the appropriate curves $C l_{\min }$ (see the lower solid and dashed lines in Figs $3 \mathrm{a}$ and $3 \mathrm{k}$ ). The same statement holds for the curves $Z_{\max }$ and $Z_{\min }$ in Figs $3 \mathrm{~b}$ and $3 \mathrm{~d}$. This serves us as a justification of using the perturbation theory.

To demonstrate the value of the deformation of the discord distribution under the small amplitude noise-polarization we represent the discord distribution in the cluster of correlated 


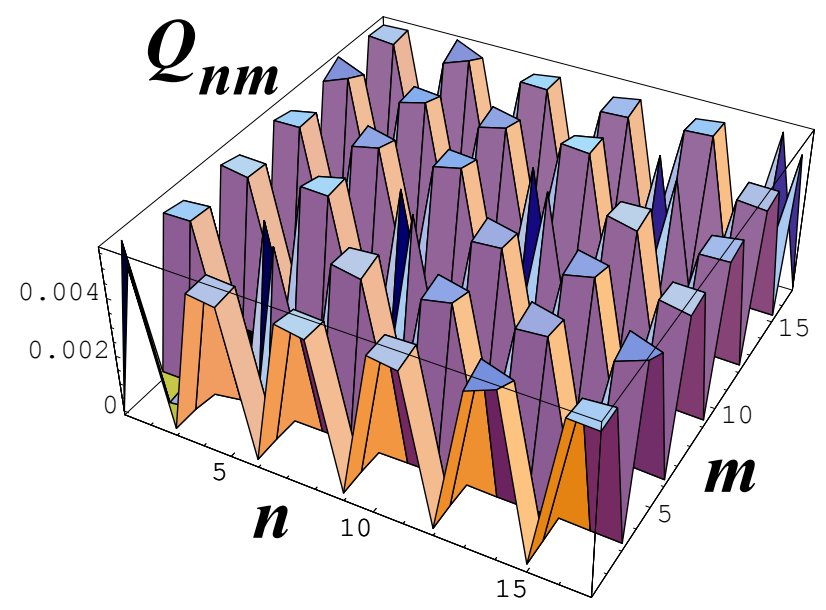

Figure 4: The averaged discord distribution $Q_{n m}$ in the system of $N=17$ fermions with the initially polarized node $j_{0}=6$, density matrix $\rho_{2}$ and noise amplitude $\epsilon=0.4$. The pairwise discord is averaged over $10^{2}$ realizations of random choices of the parameters $\tilde{b}_{j}, j=1, \ldots, 17$. This distribution is slightly deformed in comparison with that in Fig.2 for $\epsilon=0$. The difference is visible in the peaks.

fermions for the initially polarized node $j_{0}=6$, the density matrix $\rho_{2}=\frac{\tilde{\rho}_{2}}{Z_{2}}$, and the noiseamplitude $\epsilon=0.4$ in Fig, 4 . The comparison of Fig 4 with Fig 2 a shows that the deformation of the discord distribution is approximately negligible in the cluster of the correlated fermions $\mathrm{Cl}$. It is important that the noise does not destroy the stationarity of the discord distribution, similar to the parasitic polarization considered in Sec 3 ,

\subsection{Stationarity of the pairwise discord in a fermion system with noise}

In the previous section, we demonstrate that the pairwise discord in the Jordan-Wigner fermion system with a single initially polarized node remains stationary under perturbations of two types: the parasitic polarization of two neighboring nodes and the noise polarization considered by the perturbation method. In both cases, the density matrix operator involves at most threefermion terms, see eqs.(10) and (29). However, it can be readily shown that the stationarity may not be destroyed by the noise polarization even if we take into account all terms of the perturbed density matrix. Or, even more generally, the distribution of the pairwise discord is stationary for the initial density matrix of the form

$$
\begin{aligned}
& \rho_{0}=\frac{\tilde{\rho}}{Z}, \quad Z=\operatorname{Tr} \tilde{\rho} \\
& \tilde{\rho}=1+\sum_{i} \gamma_{i} I_{z i}+\sum_{i_{1} \neq i_{2}} \gamma_{i_{1} i_{2}} I_{z i_{1}} I_{z i_{2}}+\sum_{i_{1} \neq i_{2} \neq i_{3}} \gamma_{i_{1} i_{2} i_{3}} I_{z i_{1}} I_{z i_{2}} I_{z i_{3}}+\cdots+\gamma_{1 \ldots N} I_{z 1} \ldots I_{z N},
\end{aligned}
$$


where $\gamma$ 's are scalar constants. The evolution of the density matrix described by the Liouville equation $\frac{d \rho}{d t}=-i[H, \rho]$ reads:

$$
\rho(t)=e^{-i t H} \rho_{0} e^{i t H}
$$

After some transformations using equations (2), (3), (6) and (9), we obtain the density matrix in the form (we write the $t$-dependence explicitly)

$$
\begin{aligned}
\rho(t)= & \frac{1}{Z}\left(1+\sum_{i=1}^{N} \alpha_{k k^{\prime}}^{i} e^{-i t\left(\varepsilon_{k}-\varepsilon_{k^{\prime}}\right)} \beta_{k}^{+} \beta_{k^{\prime}}+\right. \\
& \left.\sum_{i_{1}, i_{2}=1}^{N} \alpha_{k_{1} k_{2} k_{1}^{\prime} k_{2}^{\prime}}^{i_{1} e_{2}} e^{-i t\left(\varepsilon_{k_{1}}+\varepsilon_{k_{2}}-\varepsilon_{k_{1}^{\prime}}-\varepsilon_{k_{2}^{\prime}}\right)} \beta_{k_{1}}^{+} \beta_{k_{1}^{\prime}} \beta_{k_{2}}^{+} \beta_{k_{2}^{\prime}}+\ldots\right),
\end{aligned}
$$

where $\alpha$ 's are expressed in terms of $\gamma^{\prime}$ s. Eq.(35) is an infinite series. An important fact of its structure is that the product of the operators $\beta_{k}^{+} \beta_{k^{\prime}}$ appears together with the exponential $e^{-i t\left(\varepsilon_{k}-\varepsilon_{k^{\prime}}\right)}$. Considering the reduced density matrix operator with respect to all fermions except for the $n$th and $m$ th ones we obtain the density matrix in the form

$$
\rho_{n m}(t)=\tilde{\alpha}_{0}^{n m}+\sum_{k, k^{\prime}=n, m} \tilde{\alpha}_{k k^{\prime}}^{n m} e^{-i t\left(\varepsilon_{k}-\varepsilon_{k}^{\prime}\right)} \beta_{k}^{+} \beta_{k^{\prime}}+\tilde{\alpha}_{n m n m}^{n m} \beta_{n}^{+} \beta_{m}^{+} \beta_{m} \beta_{n}
$$

where all the coefficients $\tilde{\alpha}$ 's are expressed in terms of the coefficients $\alpha$ 's of eq.(35) and do not depend on $t$. We do not give the explicite expressions for the $\tilde{\alpha}$ 's. Terms of higher degrees in the $\beta$-operators do not appear in the two-particle density matrix operator (36) because of the fermion operator property $\beta_{k}^{2}=\left(\beta_{k}^{+}\right)^{2}=0$. Using the basis (17), we may represent the density operator (36) in the matrix form:

$$
\rho_{n m}(t)=\left(\begin{array}{cccc}
\tilde{\alpha}_{0}^{n m} & 0 & 0 & 0 \\
0 & \tilde{\alpha}_{0}^{n m}+\tilde{\alpha}_{n n}^{n m} & e^{-i t\left(\varepsilon_{n}-\varepsilon_{m}\right)} \tilde{\alpha}_{n m}^{n m} & 0 \\
0 & e^{i t\left(\varepsilon_{n}-\varepsilon_{m}\right)}\left(\tilde{\alpha}_{n m}^{n m}\right)^{*} & \tilde{\alpha}_{0}^{n m}+\tilde{\alpha}_{m m}^{n m} & 0 \\
0 & 0 & 0 & \tilde{\alpha}_{0}^{n m}+\tilde{\alpha}_{n n}^{n m}+\tilde{\alpha}_{m m}^{n m}+\tilde{\alpha}_{n m n m}^{n m}
\end{array}\right) .
$$

Thus, the $t$-dependence appears only in the exponents in the non-diagonal elements.

Now we repeat the arguments used in the demonstration of the discord stationarity in Secs 3.1.1 and 4. Namely, it is shown in the Appendix, that the pairwise discord in X-matrix (37) depends on the absolute value $\left|\tilde{\alpha}_{n m}^{n m}\right|$ of the non-diagonal element of this matrix. Consequently the discord does not depend on the time $t$.

\section{Conclusions}

In this paper we show that the property of stationarity for the pairwise discord in the system of Jordan-Wigner fermions is stable with respect to the polarization-like perturbations of the initial state. Two types of such parasitic polarizations are considered in details. The first one 
is associated with the experimental error in the creation of the single-node polarization initial state resulting in low polarizations of the neighboring nodes. The second one is related with the noise-polarization of all nodes. The only effect of both such perturbations is deformation of the pairwise discord distribution in the cluster $\mathrm{Cl}$ of correlated fermions. In particular, such perturbations can destroy the cluster, which is explicitly demonstrated in Sec 3 for the case of two neighboring node parasitic polarization. Thus, the discord stationarity in the JordanWigner fermion system can be taken as a reliable and stable advantage of the considered fermion system in comparison with the original spin system. This encourages us to consider the possibility of a quantum gate realization on the basis of such systems of virtual particles.

This work is supported by the Program of the Presidium of RAS No.8 "Development of methods of obtaining chemical compounds and creation of new materials" and by the Russian Foundation for Basic Research, grant No.13-03-00017.

\section{Appendix: Quantum discord in the $X$-type state (37)}

The two-particle density matrix considered in this paper is a particular case of the so-called X-matrix [27]:

$$
\rho^{r e d} \equiv \rho^{(n m)}=\left(\begin{array}{cccc}
\rho_{11} & 0 & 0 & 0 \\
0 & \rho_{22} & \rho_{23} & 0 \\
0 & \rho_{23}^{*} & \rho_{33} & 0 \\
0 & 0 & 0 & \rho_{44}
\end{array}\right), \quad \sum_{i=1}^{4} \rho_{i i}=1 .
$$

The discord for the X-matrix was studied in [27]. Remind that the discord between particles $n$ and $m$ of a biparticle quantum system can be calculated as

$$
Q_{m}=\mathcal{I}(\rho)-\mathcal{C}^{m}(\rho),
$$

provided that the von Neumann type measurements are performed over the particle $m$. Here $\mathcal{I}(\rho)$ is the total mutual information [4, which may be written as follows:

$$
\mathcal{I}(\rho)=S\left(\rho^{(n)}\right)+S\left(\rho^{(m)}\right)+\sum_{j=0}^{3} \lambda_{j} \log _{2} \lambda_{j},
$$

where $\lambda_{j}(j=0,1,2,3)$ are the non-zero eigenvalues of the density matrix $\rho^{(n m)}$,

$$
\begin{aligned}
& \lambda_{0}=\rho_{11}, \quad \lambda_{1}=\rho_{44}, \\
& \lambda_{2,3}=\frac{1}{2}\left(\rho_{22}+\rho_{33} \pm \sqrt{\left(\rho_{22}-\rho_{33}\right)^{2}+4\left|\rho_{23}\right|^{2}}\right),
\end{aligned}
$$

and $\rho^{(n)}=\operatorname{Tr}_{m} \rho^{(n m)}$ and $\rho^{(m)}=\operatorname{Tr}_{n} \rho^{(n m)}$ are the marginal density matrices. The appropriate entropies $S\left(\rho^{(n)}\right)$ and $S\left(\rho^{(m)}\right)$ are given by the following formulas:

$$
\begin{aligned}
& S\left(\rho^{(n)}\right)=-\left(\rho_{11}+\rho_{22}\right) \log _{2}\left(\rho_{11}+\rho_{22}\right)-\left(\rho_{33}+\rho_{44}\right) \log _{2}\left(\rho_{33}+\rho_{44}\right), \\
& S\left(\rho^{(m)}\right)=-\left(\rho_{11}+\rho_{33}\right) \log _{2}\left(\rho_{11}+\rho_{33}\right)-\left(\rho_{22}+\rho_{44}\right) \log _{2}\left(\rho_{22}+\rho_{44}\right) .
\end{aligned}
$$


The so-called classical counterpart $\mathcal{C}^{B}\left(\rho^{(n m)}\right)$ of the mutual information can be found considering the minimization over projective measurements performed on the particle $m$ as follows [27]:

$$
\mathcal{C}^{(m)}(\rho)=S\left(\rho^{(n)}\right)-\min _{k \in[0,1]}\left(p_{0} S_{0}+p_{1} S_{1}\right)
$$

where

$$
\begin{aligned}
& S\left(\theta_{i}\right) \equiv S_{i}=-\frac{1-\theta_{i}}{2} \log _{2} \frac{1-\theta_{i}}{2}-\frac{1+\theta_{i}}{2} \log _{2} \frac{1+\theta_{i}}{2}, \\
& p_{0}=\left(\rho_{11}+\rho_{33}\right) k+\left(\rho_{22}+\rho_{44}\right) l, p_{1}=\left(\rho_{11}+\rho_{33}\right) l+\left(\rho_{22}+\rho_{44}\right) k, \\
& \theta_{0}=\frac{1}{p_{0}} \sqrt{\left(\left(\rho_{11}-\rho_{33}\right) k+\left(\rho_{22}-\rho_{44}\right) l\right)^{2}+4 k l\left|\rho_{23}\right|^{2}} \\
& \theta_{1}=\frac{1}{p_{1}} \sqrt{\left(\left(\rho_{11}-\rho_{33}\right) l+\left(\rho_{22}-\rho_{44}\right) k\right)^{2}+4 k l\left|\rho_{23}\right|^{2}} .
\end{aligned}
$$

Here, the parameters $k$ and $l$ are related by the equation [27]

$$
k+l=1
$$

It is simple to show that the quantum discord $Q_{n}$ obtained performing the von Neumann type measurements on the particle $n$ is related with $Q_{m}$ as follows:

$$
Q_{n}=\left.Q_{m}\right|_{\rho_{22} \leftrightarrow \rho_{33}}
$$

for the system with the density matrix $\rho^{\text {red }}$ given by eq.(38). Then we define the discord $Q_{n m}$ as the minimum of $Q_{n}$ and $Q_{m}[29]$

$$
Q_{n m}=\min \left(Q_{n}, Q_{m}\right), \quad n \neq m
$$

with the obvious property $Q_{n m}=Q_{m n}$. We see that, if $\rho_{n n}, n=1,2,3,4$, and $\left|\rho_{23}\right|$ do not depend on the time $t$, then the discord does not evolve with the time as well.

\section{References}

[1] Werner, R.F., Phys. Rev. A 40, 4277 (1989)

[2] W. H. Zurek, Ann. Phys.(Leipzig), 9, 855 (2000)

[3] L.Henderson and V.Vedral J.Phys.A:Math.Gen. 34, 6899 (2001)

[4] H.Ollivier and W.H.Zurek, Phys.Rev.Lett. 88, 017901 (2001)

[5] W. H. Zurek, Rev. Mod. Phys. 75, 715 (2003)

[6] L.Amico, R.Fazio, A.Osterloh and V.Vedral, Rev. Mod. Phys. 80, 517 (2008) 
[7] Horodecki, R., Horodecki, P., Horodecki, M., Horodecki, K., Rev. Mod. Phys. 81, 865 (2009)

[8] Bennett, C.H., DiVincenzo, D.P., Fuchs, C.A., Mor, T., Rains, E., Shor, P.W., Smolin, J.A., Wootters, W.K., Phys. Rev. A 59, 1070 (1999)

[9] Horodecki, M., Horodecki, P., Horodecki, R., Oppenheim, J., Sen, A., Sen, U., SynakRadtke, B., Phys. Rev. A 71, 062307 (2005)

[10] Niset, J., Cerf, N.J., Phys. Rev. A 74, 052103 (2006)

[11] Datta, A., Flammia, S.T., Caves, C.M., Phys. Rev. A 72, 042316 (2005)

[12] Datta, A., Vidal, G., Phys. Rev. A 75, 042310 (2007)

[13] Datta, A., Shaji, A., Caves, C.M., Phys. Rev. Lett. 100, 050502 (2008)

[14] Lanyon, B.P., Barbieri, M., Almeida, M.P., White, A.G., Phys. Rev. Lett. 101, 200501 (2008)

[15] J. Baum, M. Munowitz, A. N. Garroway, and A. Pines, J. Chem. Phys 83, 20015 (1985).

[16] S. I. Doronin, I. I. Maksimov and E. B. Fel'dman J. Exp. Theor. Phys. 91, 597 (2000)

[17] S.Zhang, B.H. Meier and R.R.Ernst, Phys.Rev.Lett. 69, 2149 (1992)

[18] E.B.Fel'dman, R.Brüschweiler and R.R.Ernst, Chem.Phys.Lett. 294, 297 (1998)

[19] E.B.Fel'dman and A.I. Zenchuk, Phys.Rev.A 86, 012303 (2012)

[20] P.Jordan, E.Wigner, Z.Phys. 47, 631 (1928)

[21] E. B. Fel'dman and A. I. Zenchuk, Quantum Inf. Process. 13, 201 (2014)

[22] G. De Chiara, D. Rossini, S. Montangero, R. Fazio, Phys. Rev. A 72, 012323 (2005)

[23] A. Zwick, G.A. Álvarez, J. Stolze, O. Osenda, Phys. Rev. A 84, 022311 (2011)

[24] 11. A. Zwick, G.A. Álvarez, J. Stolze, O. Osenda, Phys. Rev. A 85, 012318 (2012)

[25] A. Zwick, G.A. Álvarez, J. Stolze, O Osenda, arXiv preprint arXiv:1306.1695, (2013)

[26] J.Stolze, G. A. Álvarez, O. Osenda, A. Zwick in Quantum State Transfer and Network Engineering. Quantum Science and Technology, ed. by G.M.Nikolopoulos and I.Jex, Springer Berlin Heidelberg, Berlin, p.149 (2014)

[27] M.Ali, A.R.P.Rau, G.Alber, Phys.Rev.A 81, 042105 (2010); Erratum: Phys.Rev.A 82, 069902(E) (2010) 
[28] L. D. Landau and E. M. Lifshitz, Course of Theoretical Physics, Vol. 3: Quantum Mechanics: Non-Relativistic Theory (Nauka, Moscow, 1974; Pergamon, New York, 1977).

[29] E.B.Fel'dman and A.I.Zenchuk, JETP Lett. 93, 459 (2011) 